\title{
Modernidad e internacionalización: Arte en Colombia (1943-1956)
}

\section{Modernity and Internationalization: Art in Colombia (1943-1956)}

\author{
Alexandra Mesa Mendieta \\ Universidad Autónoma de Madrid, España. \\ arrasana@gmail.com
}

\section{Resumen}

Entre 1943 y 1956 artistas colombianos fueron invitados a participar de eventos de exposición en el extranjero. Tres de ellos, que revelan dinámicas para el arte occidental, guían la estructura de este artículo: la exposición de la colección de arte latinoamericano del MoMA (1943), 32 artistas de las Américas (1949) y la Bienal Hispanoamericana de Arte (1951-1956). La investigación da cuenta de este período de movilidad artística y sus efectos, apoyándose en bibliografía secundaria y en información consignada en documentos pertenecientes al Museo Nacional de Colombia, el Museo de Arte de las Américas, el MoMA y el Museo Nacional Centro de Arte Reina Sofía.

Palabras clave: arte moderno, arte colombiano, coleccionismo, arte latinoamericano, Bienal Hispanoamericana de Arte.

\section{Abstract}

Colombian artists were invited to participate in exhibition events abroad between 1943 and 1956. Three of them, which reveal dynamics for Western art, guide the structure of this article: The Latin-American Collection of the Museum of Modern Art (1943), 32 artistas de las Américas (1949) and the Hispano-American Biennial of Art (1951-1956). The research analyzes this period of artistic mobility and its effects, relying on secondary sources and information contained in documents belonging to the Museo Nacional de Colombia, the Museo de Arte de las Américas, The Museum of Modern Art (MoMA) and the Museo Nacional Centro de Arte Reina Sofía.

Keywords: modern art, Colombian art, collecting, Latin-American art, Hispano-American Biennial of Art. 


\section{Introducción}

Entre 1955 y 1956, el artista Alejandro Obregón (Barcelona, España, 1925-Cartagena, Colombia, 1991) tuvo una exposición monográfica en la sala de la Organización de Estados Americanos (OEA) en Washington; una de sus obras ingresó a la colección de The Museum of Modern Art (MoMA) de Nueva York y fue premiado en al menos tres certámenes de corte internacional: la Bienal Hispanoamericana de Arte, el Gulf Caribbean Competition y los premios Guggenheim. En ese mismo momento, el Museo Nacional de Colombia adquirió por primera vez una de sus pinturas, Máscaras (1952) (fig. 1).

En este óleo de fondo verde se reconoce una figura femenina de cuerpo entero con una máscara indígena, que en una bandeja ofrece alimentos junto a un yelmo de conquistador unido a una máscara antigás. Los objetos representados en la pintura, sobre todo las máscaras, proponen una reflexión simbólica frente a procesos históricos de colonización. La obra habría sido pintada en Francia a siete años del fin de la Segunda Guerra Mundial, en un contexto tenso de polarización global y en uno de los momentos de violencia política más críticos en la historia de Colombia.

Máscaras pronto fue expuesta en el edificio panóptico donde ocho años atrás se había instalado de manera definitiva el Museo Nacional. La pintura se ubicó en una pared de la escalera que separaba la colección histórica del museo ubicada en el segundo piso, de la colección de bellas artes dispuesta en el tercero. Este lienzo contrastaba con las otras piezas históricas y artísticas con las que se tejía la versión oficial de la identidad colombiana.

La presencia de la "mujer sobre fondo verde" confrontaba al historicismo pictórico, tal como lo expresó Marta Traba en un artículo llamado "Las generaciones de la escalinata”, publicado en el periódico El Tiempo el 7 de julio de 1957. La afamada crítica de arte argentina, radicada en Colombia desde 1954, hizo evidente el carácter modernista de Máscaras al comparar sus características formales con las de La Mujer de Sacala de Víctor Mideros (ca. 1940), una valiosa pintura ecuatoriana de carácter figurativo, que se encontraba expuesta cerca. ${ }^{1}$

No hay certeza de cuánto tiempo estuvo exhibida la pintura de Obregón en esta ocasión, sin embargo, esta adquisición y su ágil puesta en escena merecen ser pensadas a la hora de estudiar la inclusión de lenguajes modernistas en los circuitos artísticos bogotanos. Vale la pena recordar que en la década de 1950, el Museo Nacional era el único espacio público en la capital colombiana que exponía de manera abierta y permanente una colección con obras de artistas vivos.

1 Esta pintura estuvo catalogada por años como La mujer de Salazaca y en su artículo Marta Traba se refiere a La Mujer de Sacala. Sin embargo, en 2003, la historiadora Alexandra Kennedy Troya aclaró que el personaje representado es un hombre. Salazaca hace referencia a una tribu indígena ecuatoriana. Hoy esta pintura está referenciada como Hombre Salazaca (Gonzáles y Lleras 174). 


\section{FIGURA 1}

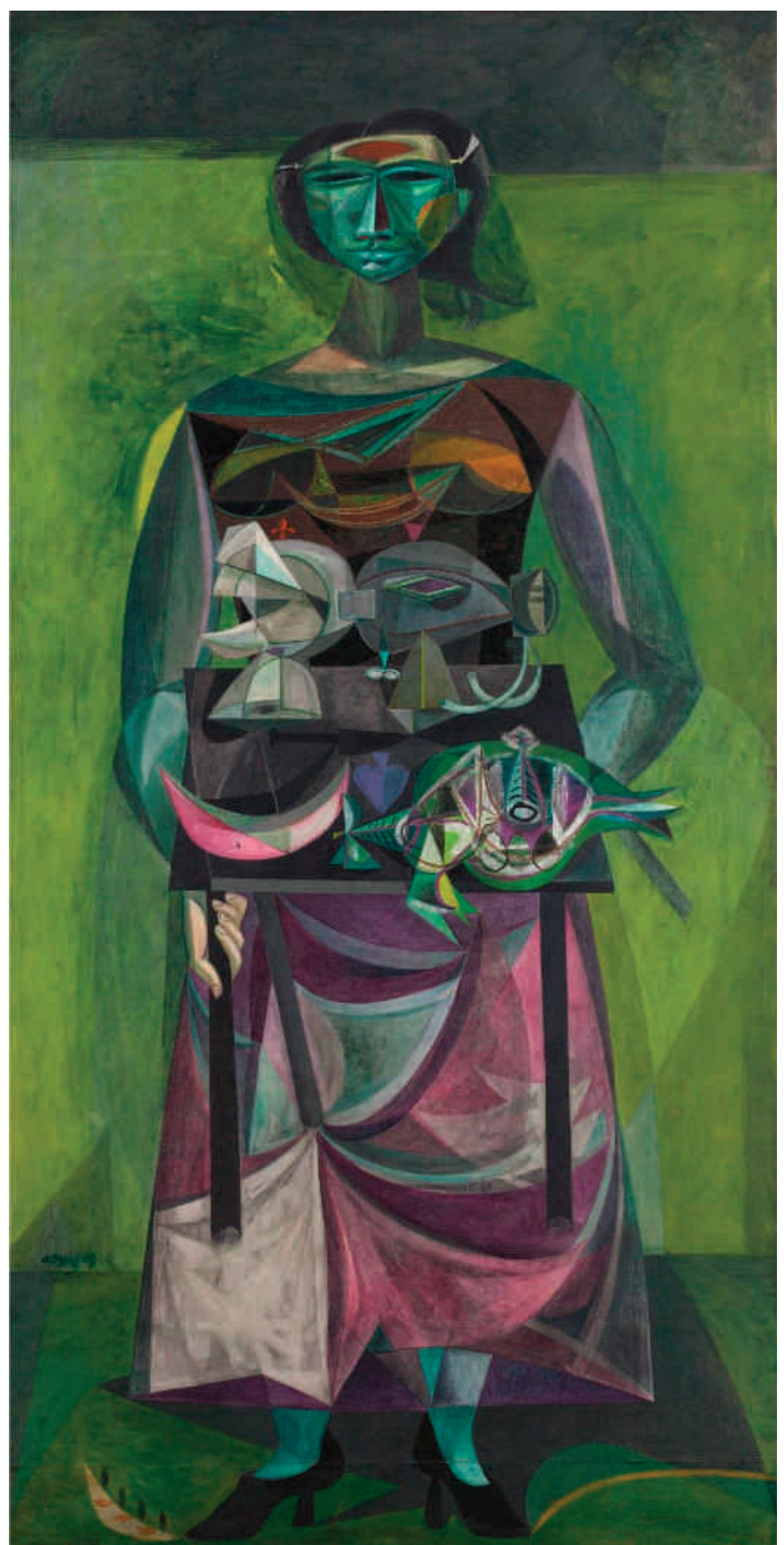

Alejandro Obregón, Máscaras (ca. 1952). Óleo sobre tela, 210 x 107 cm. Colección Museo Nacional de Colombia, reg. 2219. Reproducción fotográfica: @Museo Nacional de Colombia. 
El propósito de este artículo es exponer el contexto en el que una pintura como Máscaras, explícitamente moderna según la crítica especializada de la época, ingresa a una colección pública de arte en Colombia. Dos preguntas atraviesan este análisis: ¿Qué tipo de obras fueron consideradas modernas en Colombia a mitad del siglo xx? $\mathrm{Y}$ ¿cómo estas piezas artísticas transitan hasta convertirse en objetos simbólicos de interés patrimonial coleccionados por un museo?

Sin duda, la obra de arte (moderno) juega un papel fundamental como unidad de análisis a la hora de estudiar los procesos artísticos en Colombia a través del siglo $\mathrm{xx}$, teniendo en cuenta que funciona como un objeto material y simbólico mutable a partir del que se puede entender la construcción de identidades tanto estéticas como culturales. Es un instrumento portador de legitimidades y diferencias que además de su significado creativo, adquiere valor en contextos de circulación y coleccionismo. De acuerdo con Marcelo Pacheco, la observación de estos contextos de acción -locales, regionales, globales- permite entender una historia cruzada por tensiones y encuentros entre diferentes variables y operadores (100).

La creación de Máscaras y el contexto que la avala como una pintura moderna, dan cuenta de un momento en que el arte colombiano asume una modernidad de corte universalista, definida en gran parte a partir de dinámicas externas, relacionadas con hechos como el ascenso del MoMA como autoridad para el arte occidental durante la primera mitad del siglo xx, e influida por el reordenamiento de la geopolítica durante y después de la Segunda Guerra Mundial, cuando las potencias antiguas y las nuevas procuran ampliar su influencia cultural alrededor del globo, con el fin de ejercer poder político y económico. Tiempo también en el que, en respuesta a las atrocidades de la guerra, se promueve la convivencia pacífica de las naciones, facilitando el intercambio y aportando a la institucionalización de la cultura, asunto evidente en la fundación de entes especializados en la promoción de las artes y las letras, y de organizaciones internacionales como la Organización de las Naciones Unidas para la Educación, la Ciencia y la Cultura (UNESCO) y el Consejo Internacional de Museos (ICOM), como una de sus dependencias.

En este contexto, durante las décadas de 1940 y 1950 se gestionan colecciones de arte y se realizan concursos y exposiciones de carácter internacional -promovidas desde países como España y Estados Unidos- en los que participan obras colombianas, procurando prestigio a sus artífices y acrecentando el valor de su trabajo. Muchos de estos eventos justificados en afinidades culturales y geográficas acogen la producción plástica de los países de la América Latina, proponiendo amplias comunidades en pro del panamericanismo o el hispanoamericanismo, según el caso. Desde institutos de cultura, museos y organizaciones internacionales se realizan eventos como: la exposición de la colección de arte latinoamericano del MoMA en 1943; 32 artistas de las Américas, patrocinada por la OEA con el apoyo del MoMA en 1949, y la Bienal Hispanoamericana de Arte, financiada por el gobierno franquista y organizada por el Instituto de Cultura Hispánica (1951, 1954, 1955). 
Estas acciones globales en pro de la internacionalización, desde diferentes perspectivas y contextos, poco a poco respaldaron una estética que, a pesar de las diferencias en la concepción y ejecución de las obras, pretendía determinar un lenguaje común de lectura universal para ellas, como apuntó Alfred H. Barr, Jr. en 1943 en su introducción a What is Modern Painting?:

Some pictures are easy, like a primer, and some are hard with long words and complex ideas; and some are prose, others are poetry, and others still are like algebra or geometry. But one thing is easy, there are no foreign languages in painting as there are in speech; there are only local dialects which can be understood internationally, for painting is a kind of visual Esperanto (3).

Al tomar partido frente a esta pretendida universalización, se estimuló una transformación continua de las narrativas para las historias del arte y constantes cuestionamientos frente a lo que podía considerarse como una obra de arte moderno. En este sentido, el análisis que se propone en este texto comulga con la idea del curador venezolano Luis Pérez Oramas, quien expresa que el arte moderno no es una categoría rígida, unívoca ni parcial, más bien funciona como un asunto dinámico, complejo y que adquiere sentido al relacionar eventos, lenguajes y geografías:

Because we believe that modern art is not solely a history of prominent figures and individual names, a sum of geniuses. On the contrary, if anything distinguishes the modern interpretation of meaning, it is formation of structural synchronies: The idea that signs, icons, symbols, styles, texts, visual forms, and works of art are dynamic elements subject to constant transformation and only make sense through comparison, analogy, repetition, and differentiation among themselves (38).

El proceso de definición de la modernidad artística para el caso colombiano, a partir de criterios de internacionalización a mediados del siglo xx, puede pensarse a partir de 1943, cuando obras de artistas nacionales fueron incluidas en la recién conformada colección de arte latinoamericano del MoMA. Tiene un primer cierre visible cuando, más de una década después, una modernidad internacional que rompía con la figuración tradicional fue respaldada por una naciente crítica de arte especializada, y empezó a ser asimilada de manera permanente por los espacios de arte local mediante prácticas de circulación como el coleccionismo público, asunto que podemos ubicar entre 1956 y 1963.

Como se ha anotado antes, fue justo en 1956 cuando el Museo Nacional pagó mil quinientos pesos a Alejandro Obregón por una de sus pinturas para pronto exponerla de manera amplia, como representación de la modernidad artística y como patrimonio. Y fue en 1963 cuando, tras varios intentos, finalmente se instaló de manera permanente y con una colección propia un museo de arte moderno en Bogotá.

El presente artículo se divide en tres partes que dan cuenta de la participación de artistas colombianos en exposiciones que, en un contexto de internacionaliza- 
ción, los avalaron como modernos y cuyo análisis permite comprender los cambios en los circuitos y en los lenguajes artísticos locales en el período entre 1943 y 1956. El apartado inicial está dedicado a la participación nacional en la exposición de la primera versión de la colección de arte latinoamericano del MoMA en 1943. El siguiente se enfoca en la exhibición 32 artistas de las Américas en el Museo Nacional en 1949, evento que inauguró las acciones hacia Colombia que en cuestiones artísticas adelantó el Departamento de Artes Visuales de la OEA a mediados del siglo xx. Y en el tercero se estudian los procesos de internacionalización del arte local durante el período comprendido entre 1951 y 1956, utilizando como unidad de análisis los envíos colombianos a las tres versiones de la Bienal Hispanoamericana de Arte realizadas en Madrid, La Habana y Barcelona, y los gestos de oposición frente a un evento organizado por un gobierno totalitario.

Las tres partes se enmarcan en períodos precisos (1943-1948, 1948-1951 y 1951-1956). Por estar ordenadas cronológicamente podría pensarse que responden a procesos consecutivos, pero en realidad responden a procesos simultáneos de los que se han elegido momentos puntuales, teniendo en cuenta su relevancia. Cada apartado proyecta asuntos que sobrepasan la temporalidad de los eventos de exposición descritos y propone líneas de largo aliento visibles durante el período de la Guerra Fría, como la incidencia de la hegemonía cultural estadounidense en la escena artística colombiana, la cuestionada intervención del Departamento de Artes Visuales de la OEA en la construcción de una red institucional para el arte moderno en América Latina y la construcción de comunidades de naciones a partir de afinidades culturales y geográficas tras la Segunda Guerra Mundial, respaldándose en el arte moderno y su concebida universalización.

Este panorama de los eventos internacionales durante las décadas de los cuarenta y cincuenta, y su incidencia en los discursos creativos y en el perfil del artista moderno, más que ofrecer una descripción historicista, invita a generar matices y complejizar el entramado de los escenarios artísticos en Colombia a mitad del siglo pasado y a comprender cómo las dinámicas internacionales influyeron en la manera en que se expuso y coleccionó arte moderno desde organizaciones locales con vocación pública.

\section{Artistas colombianos en la colección del MoMA (1943-1948)}

Ya en 1943, el Museo de Arte Moderno (de Nueva York) se había convertido en la institución que hoy conocemos. Incluso entonces, su colección permanente era considerada como la más importante del mundo dentro de su género; sus exposiciones - muchas de las cuales viajaban a través de su departamento de Exposiciones- poseían justo renombre; las publicaciones que las acompañaban destacaban por su erudición, lucidez y calidad de diseño; los departamentos de Arquitectura y Fotografía del Museo se hallaban ya constituidos, así como 
un programa en expansión de diseño industrial; funcionaban una filmoteca y una biblioteca cuya colección de arte moderno pronto se consideraría sin rival; se había iniciado un programa internacional; se había fundado una galería para jóvenes, se publicaba un boletín y, desde 1939, el Museo se alojaba en un nuevo y moderno edificio de West 53rd Street. Todo ello se había logrado bajo la dirección de Barr (Sandler 30).

En este párrafo extraído de la introducción al libro La definición del arte moderno, donde se recopilan importantes documentos de la producción escrita de Alfred Barr, se describe cómo era el MoMA en 1943, justo en el momento en el que el museo neoyorquino configura y expone por primera vez una colección de arte latinoamericano. Un museo con una perspectiva amplia respecto a la modernidad y que en teoría respaldaba la universalización de los lenguajes plásticos, de repente, a partir de un criterio geográfico (Latin-American Art), incluía en su colección una cantidad considerable de piezas de arte. ${ }^{2}$

El proceso de concreción de una colección de arte latinoamericano para el MoMA y su incidencia para la historia del arte de Occidente ha sido estudiado por historiadores como Miriam Basilio, Luis Pérez Oramas, Eustáquio Ornelas Cota y Beverly Adams, quienes concuerdan en que uno de los factores que posibilitó esta empresa fue la creación en 1942 de un fondo interamericano para adquisiciones, promovido por Nelson Rockefeller. Este gesto estuvo enmarcado en una serie de políticas de "buena vecindad" asumida por los Estados Unidos durante las décadas de 1940 y 1950, que por supuesto también beneficiaba a los propietarios de grandes capitales, muchos de ellos con inversiones en América del Sur y algunos benefactores del museo.

El propósito de enfocar la geopolítica mundial hacia el oeste del Atlántico tras el ascenso de los Estados Unidos como potencia, se hizo evidente y condujo a acciones para el autorreconocimiento de América, acentuando su participación en la cultura occidental. En la presentación al catálogo The Latin-American Collection of the Museum of Modern Art, escrita por Alfred Barr, se puede leer: "Thanks to the Second World War and to certain men of good will throughout our Western Hemisphere, we are dropping those blinders in cultural understanding which have kept the eyes of all the American republics fixed on Europe with scarcely a side glance at each other during the past century and a half" (3).

Tanto desde el ámbito público como desde el privado se convocaba a una comunidad panamericanista amplia. El ingreso de dinero al Fondo Interamericano proveniente de donaciones -algunas anónimas- estimuló el viaje de Alfred Barr, director y curador del MoMA, y de Lincoln Kirstein, uno de sus asesores, quienes

2 Aunque en este artículo no se profundizará en las tensiones que implica para la plástica moderna esta categoría de "arte latinoamericano", vale la pena revisar las investigaciones al respecto de autores como Marta Traba y, más recientemente, Luis Pérez Oramas y Luis Camnitzer. 
con el propósito de estudiar los procesos artísticos de la América Latina y hacerse de obras relevantes, emprendieron un recorrido por el continente. Alfred Barr estuvo en México y Cuba, y Lincoln Kirstein se hizo cargo de América del Sur (Brasil, Argentina, Uruguay, Chile, Ecuador, Perú y Colombia).

Como resultado de esta empresa, ingresaron a la colección permanente del museo al menos 18 pinturas y acuarelas, 17 dibujos, tres esculturas, 65 grabados y varios carteles (Barr, "Foreword" 4). Al poco tiempo (entre marzo y junio de 1943), se presentó una exposición y se publicó un extenso catálogo editado por Lincoln Kirstein, en el que se daba cuenta de las nuevas adquisiciones, que, junto a cerca de ochenta obras que ya se encontraban en el acervo, conformaron la primera versión de la colección de arte latinoamericano del MoMA. Según anota Alfred Barr, se pretendía que este documento en el que se referenciaba "la colección de arte latinoamericano más importante del mundo", funcionara como un suplemento del catálogo de pintura y escultura del museo que se había publicado unos meses antes: "[...] this is a record of the most important collection of contemporary Latin-American art in the United States, or for that matter in the world (including our sister republics to the south). As such it is a supplement to the catalog, Painting and Sculpture in the Museum of Modern Art (1942), which is devoted for the most part to the art of Europe and the United States" ("Foreword" 3).

Aunque era extraño que un museo que abogaba por la universalidad de los lenguajes artísticos acudiera a una categoría geográfica para caracterizar un tipo de arte, desde 1943 el MoMA cuenta con una colección de arte específicamente latinoamericano, que en principio quizás se configuró siguiendo la pauta de escuelas y movimientos sintetizada en What is Modern Painting? (1943). En este breve libro que había sido publicado por el museo recientemente como guía ilustrativa, se mapeaba la modernidad artística abanderada por el MoMA, buscando elementos comunes y agrupando obras sin reparar en el origen de sus artífices y privilegiando la creación individual sobre los nacionalismos.

En What is Modern Painting?, desde una óptica formal, junto al surrealismo, el expresionismo, el cubismo, etcétera, se contemplaban los realismos y dentro de ellos el retrato e incluso el paisaje. Esto puede explicar por qué en la selección de Lincoln Kirstein Colombia estaría representada por siete obras, todas ellas de carácter figurativo: un autorretrato, cuatro paisajes y dos vistas populares. Un poco por fuera del formalismo artístico que pese a su amplitud se insinuaba en el libro de Barr, la selección de arte latinoamericano expuesta en 1943 tenía un matiz particular, dando un lugar visible a la pintura ingenua y folclórica.

Los cuatro artistas colombianos y sus obras habrían sido elegidos tras una aproximación a la escena local que, según la correspondencia de Kirstein consignada en el archivo histórico del museo neoyorquino, duró más o menos un año. Los trabajos de los colombianos Luis Alberto Acuña, Gonzalo Ariza, Erwin Kraus y Alfonso Ramírez Fajardo estuvieron expuestos junto a obras de Diego Rivera, José Clemente 
FIGURA 2

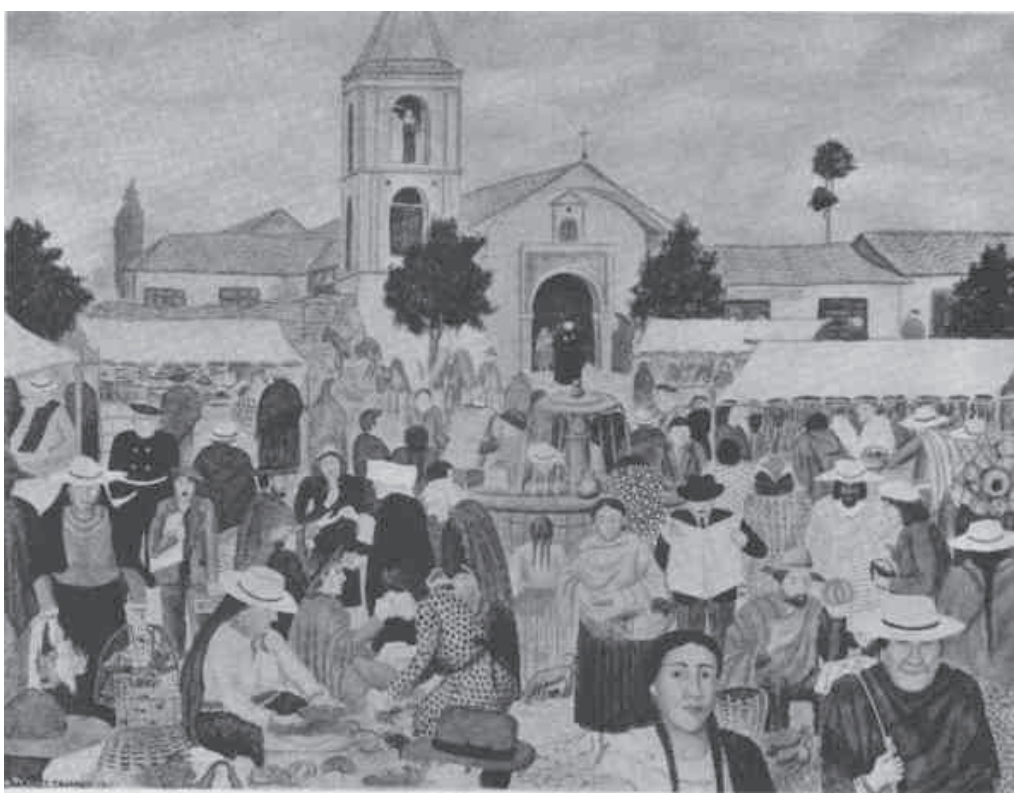

Alfonso Ramírez Fajardo, Fiesta (1942). Acuarela sobre papel, $18^{1 / 2}$ x $24^{1 / 4} 4^{\prime \prime}$. Reproducción fotográfica en blanco y negro (Kirstein 45).

\section{FIGURA 3}

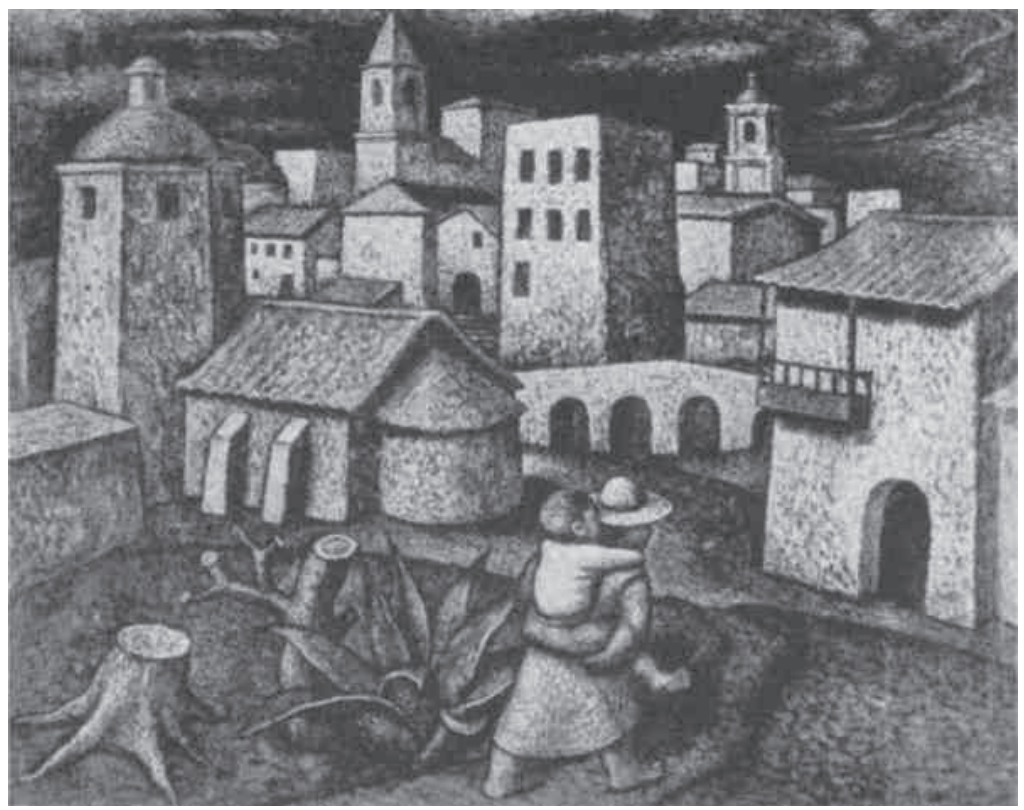

Luis Alberto Acuña, The Golden City (1942). Óleo sobre tela, $27^{3 / 8}$ x $35^{\prime \prime}$. Reproducción fotográfica en blanco y negro (Kirstein 46). 


\section{FIGURA 4}

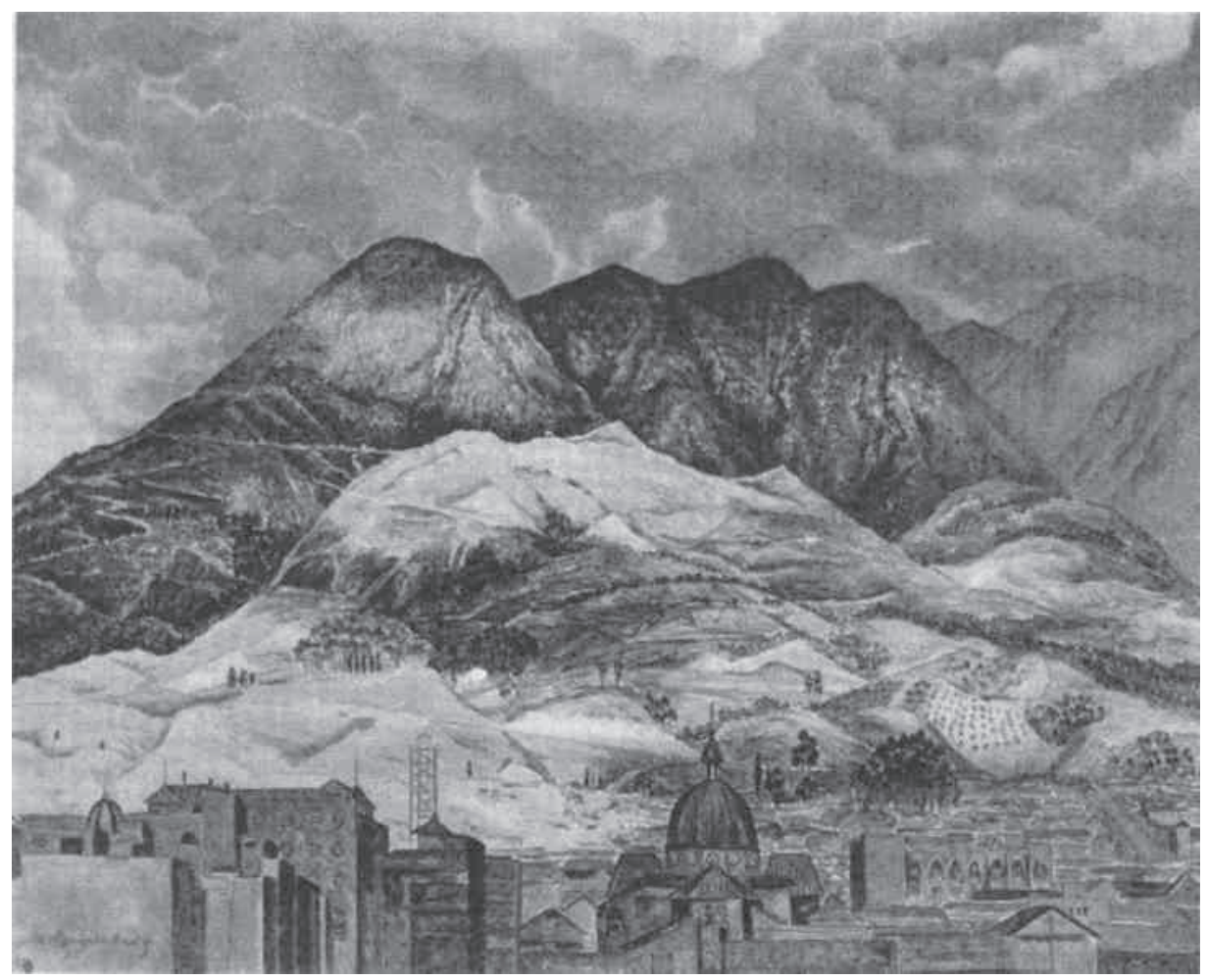

Gonzalo Ariza, Bogotá (1941). Óleo sobre tela, $31^{3 / 8}$ x $39^{1 / 2 "}$. Reproducción fotográfica en blanco y negro (Kirstein 46).

Orozco, David Alfaro Siqueiros, Emilio Pettoruti, Cândido Portinari, Roberto Matta, Amelia Peláez, Wifredo Lam y Joaquín Torres García, entre otros. Multiplicidad de propuestas y estilos se reunían en esta exposición y su selección que -se argumentaba en el prominente catálogo ilustrado- se dividía en fragmentos que caracterizaban la escena de cada país y su historia, distinto a What is Modern Painting?, estructurado a partir de escuelas y movimientos.

Además de textos escritos por Alfred Barr y Lincoln Kirstein, en el catálogo de la exhibición se imprimieron fotografías de varias de las obras (figs. 2, 3, 4); biografías de los artistas; fichas de referencia y una bibliografía extensa, aunque en algunos casos, como el de Colombia, poco detallada. ${ }^{3}$

En los textos introductorios del catálogo se aprecia el interés por repensar la tradicional historia del arte enfocada en la producción europea para dar espacio a

3 Un amplio análisis de este catálogo ha sido desarrollado por Eustáquio Ornelas Cota en su tesis de maestría en Historia social de la Universidad de São Paulo en 2016. 
América entera. Para vincular el norte y el sur del continente se acudió a afinidades derivadas del pasado colonial; es así como Lincoln Kirstein, al pensar los escenarios particulares y vincular el arte moderno de América a la historia de la cultura occidental, procura un recorrido que se remonta a los procesos de conquista hasta llegar a las influencias vanguardistas de finales del siglo xIX y las primeras décadas del Xx. En la solapa de la portada del catálogo podemos leer:

Lincoln Kirstein's concise informative text examines the cultural circumstances which have shaped the art of Latin-American countries, revealing the story of the colonial powers, the patronage of the church and viceregal courts, and the rise of national spirit and local salons with the ultimate political independence of 19th century. Many illuminating comparisons and contrasts are drawn between the art of our own country and that of the Latin-American republics, which frequently provide a double insight to the culture of the Americas as a whole. The nourishing influence of the older continent of Europe is carefully followed from the imitators of Murillo, the pupils of David, to the more recent repercussions of Impressionism and the School of Paris.

Para el caso de Colombia, Lincoln Kirstein afirma que el arte moderno puede ser pensado a partir de 1910, ubicando la producción artística inspirada en los eventos de celebración del centenario de la independencia. Luego, hace énfasis en la influencia española tras la Primera Guerra Mundial, ofrece crédito como representantes de la pintura de género a artistas vinculados a la Academia y la Escuela de Bellas Artes, como Francisco Cano, Coriolano Leudo, Roberto Pizano y Ricardo Gómez Campuzano. Finalmente, identifica un punto de quiebre hacia la modernidad de corte vanguardista, inspirado en la escena mexicana en la década de los treinta, y termina describiendo el trabajo de los artistas seleccionados por él para hacer parte de la colección de arte latinoamericano del MoMA:

By 1930 Parisian influence created a normal reaction against the previous naturalism. Jorge Zalamea, a journalist, led the new pictorial movement, affected somewhat by Mexico, but such influential artists as Ignacio Gómez Jaramillo and Pedro Nel Gómez are more academic.

A younger generation is now in healthy opposition. Luis Alberto Acuna, trained as a sculptor, shows strong plastic tendencies and some effect of Mexico, where for three years he occupied a diplomatic post [...]. Gonzalo Ariza, who had a scholarship for work in Japan, shows small Japanese influence, but has in the company of Erwin Kraus made a conscious re-discovery of the wonderful savannas around Bogotá [...]. Ramírez Fajardo is an untaught artist with close knowledge of the popular background (45).

La visita de Lincoln Kirstein a Colombia contó con el apoyo de la delegación diplomática de los Estados Unidos representada por Herschell Brickell y la selección de 
obras se apoyó en la comunicación postal del emisario del MoMA con los artistas (en especial con Erwin Kraus), quienes le enviaron información y material, como los catálogos de las ediciones II y III (1941 y 1942) del Salón Anual de Artistas (Lincoln Kirstein Correspondence and Notes). Otro punto de referencia para Kirstein fue el libro Nueve artistas colombianos, publicado en 1941. Tal como se evidencia en este libro escrito por el crítico Jorge Zalamea, y como se haría explícito en los catálogos del Salón Nacional, a principios de los años cuarenta la escena artística colombiana expresaba una multiplicidad de puntos de vista e influencias. Respecto de su selección de artistas Jorge Zalamea anotaba: "Los nombres escogidos para figurar en esta antología gráfica, ni son pares, ni significan límites de jerarquía, ni siquiera son todos de la predilección de quien les diera cita en estas páginas: desigual el valor de sus obras, diversas las normas aceptadas, contradictorio el espíritu que las anima, ofrecen sin embargo en su conjunto, una imagen casi cabal de nuestro movimiento artístico contemporáneo, en lo que él tiene de más independiente y puro" (13-14).

Dos de los nueve artistas reseñados por Jorge Zalamea fueron elegidos por Lincoln Kirstein: Luis Alberto Acuña y Gonzalo Ariza. ${ }^{4}$ En sintonía con el crítico colombiano, Lincoln Kirstein también argumentaba su elección en la independencia de la academia y el costumbrismo, para luego proponer al paisaje como el género que posibilitaba la libertad artística en el país: "The Colombian landscape is so impressive that it is heartening to find young painters, separated from both Paris and Mexico, investigating it rather than imitating a bohemian academy or supporting neo-Indian exoticism" (45).

Es de considerar que, sin negar el valor de los artistas seleccionados, la investigación de Kirstein era conservadora, rápida y genérica, además se hacía evidente el propósito de restar relevancia a la influencia mexicana caracterizándola como pasada, para dar camino a tendencias que, a su parecer, contaban con más argumentos formales (el paisaje).

A excepción de las obras de Luis Alberto Acuña - un autorretrato y una vista típica denominada Ciudad de oro, ambos de 1941 y que ingresan como donación y comodato, respectivamente-, las obras de los otros colombianos fueron adquiridas por el museo neoyorquino por medio del Fondo Interamericano.

En las reseñas bibliográficas de los artistas colombianos se describen brevemente sus carreras y se destaca su bagaje internacional, al que se atribuyen algunas de las características de su trabajo. En el caso de Alfonso Ramírez Fajardo, teniendo en cuenta su juventud, se reseña su formación autodidacta y la ingenuidad de su obra, además de su participación en el Primer Salón Nacional de Artistas Colombianos,

4 Los artistas seleccionados por Jorge Zalamea para Nueve artistas colombianos serían: Luis Alberto Acuña (1904-1984), Gonzalo Ariza (1912-1995), Ramón Barba (1892-1964), Carlos Reyes (n. 1907-?) Sergio Trujillo (1911-1999), Pedro Nel Gómez (1899-1984), Ignacio Gómez Jaramillo (1910-1970), José Domingo Rodríguez (1895?-1968) y Josefina Albarracín (1910?-2007). Esta selección hacía evidente un panorama del todo heterogéneo, que daba cuenta del punto de vista del autor expuesto a partir de un testimonio gráfico de lo que consideraba como "pintura y escultura nuevas", y en el que se unificaba la selección abogando por la independencia estética que representaban los artistas. 
evento del que también habrían hecho parte sus compañeros y del que Jorge Zalamea habría sido jurado en 1940.

Estar representados en la colección del MoMA, para ese momento la institución museística más importante en lo referente a arte moderno, y su constante participación en salones y exposiciones nacionales, garantizó el reconocimiento internacional como artistas modernos a Gonzalo Ariza, Luis Alberto Acuña y Erwin Kraus, siendo menos visible Alfonso Ramírez Fajardo, quizás por trabajar un poco alejado de los circuitos.

Para 1945, Teresa Cuervo Borda se desempeñaba como directora del Museo de Arte Colonial en Bogotá y, en enero de ese año, recibió una carta del MoMA requiriendo su colaboración en el envío de grabados para ser exhibidos en una exposición de arte gráfico latinoamericano preparada por Mrs. Sherman P. Haight del American Institute of Graphic Arts:

Como se trata sólo de arte moderno, hemos pensado en Acuña, Ariza, Kraus, etc. De Acuña estoy seguro que ha hecho litografías. ¿Podrías enterarte tú si tienen algo que quisieran mandar a la exposición? [...] La exposición está organizada conjuntamente por el American Institute of Graphic Arts y el Grolier Club. Nosotros solamente les aconsejamos de una manera extraoficial en la selección de los grabados. [...] Ojalá se decidan a mandar algo. La exposición es seria y van a estar representados los principales grupos artísticos de América Latina (AHMNC vol. 15 1945, fol. 4).

En sintonía con este eco de modernidad proveniente del MoMA que avalaba a Gonzalo Ariza, Erwin Kraus, Alfonso Ramírez Fajardo y Luis Alberto Acuña, estos fueron ampliamente valorados cuando en 1948, bajo la dirección de Teresa Cuervo, el Museo Nacional se trasladó al edificio Panóptico. Allí se reunió una colección pública de arte colombiano que daba cuenta de la historia de la era republicana desde la independencia hasta el presente. La presencia de las obras de Ariza, Acuña, Kraus y Ramírez Fajardo fue requerida, algunas de ellas fueron compradas pronto, a pesar de las restricciones presupuestarias, y otras, que ya habían sido adquiridas por el Ministerio de Educación sin un destino fijo, fueron trasladadas.

La transferencia al Panóptico de obras de otros artistas, incluso más cercanos a lenguajes de vanguardia y que habían sido partícipes de los salones nacionales, también fue solicitada y ejecutada. Este fue el caso de piezas de Guillermo Wiedemann, Enrique Grau, Omar Rayo y Hernando Tejada, quienes se desmarcaban más de la figuración tradicional que los que en ese momento se reconocían como artistas modernos fuera del país. Otros que ya comenzaban a destacar en los salones nacionales por su ímpetu moderno, como Alejandro Obregón, Édgar Negret, Eduardo Ramírez, tuvieron que esperar cerca de una década para que sus obras fueran consideradas como piezas de colección para el Museo Nacional (Mesa, ¿Identidad o modernidad?). 


\section{2 artistas de las Américas: La incursión de la OEA en la escena del arte colombiano (1948-1951)}

El panorama de internacionalización para el arte moderno colombiano vinculado a los escenarios del arte estadounidense, que se había empezado a perfilar con la participación en la exposición The Latin-American Collection of the Museum of Modern Art en 1943, tuvo un giro significativo entre 1948 y 1951, cuando el Departamento de Artes Visuales de la OEA, abanderando una identidad panamericanista, comenzó a influir en los circuitos artísticos.

En abril de 1948, justo en uno de los momentos de violencia política más álgidos en la historia de Colombia, se celebró en Bogotá la IX Conferencia Panamericana. Allí se acordó y firmó la creación de la OEA, como proyección de la Unión Panamericana fundada en 1910, con el propósito de velar por las buenas relaciones entre los países del continente. Inicialmente la Unión Panamericana fue una entidad con fines comerciales y diplomáticos y con el paso tiempo su espectro se fue ampliando; para 1948 ya intervenía de manera amplia en otras esferas, que incluían el apoyo y la difusión cultural y artística.

La renovada organización de los proyectos culturales y específicamente en lo referido a artes plásticas, apuntaba a dar forma a una escena latinoamericana gestionada desde Washington, que engranara con lo que pasaba en el norte del continente.

La exposición 32 artistas de las Américas fue la primera en Colombia que representó el origen de un entramado para el arte latinoamericano gestionado en teoría desde la OEA, impulsando la "pluralidad de tendencias artísticas de corte más subjetivo, formalista, lírico y despolitizado", dejando atrás los temas folclóricos miméticos (Armato 91-92). Este argumento ya había sido sugerido por Lincoln Kirstein y gradualmente buscaba marginar al realismo socialista que ya se sentía como un enemigo discursivo en plena Guerra Fría. Paradójicamente, para esta exposición los realistas mexicanos eran protagonistas indiscutibles a la hora de imaginar un arte de América Latina. Una evidencia fue la manera en que desde el Museo Nacional se solicitaba difundir el evento por medio de la prensa local: "El próximo lunes se abrirá también la Exposición de Pintura de los 32 Pintores de América, patrocinada por la Unión Panamericana. Entre otros figuran Rivera, Orozco, Tamayo, Siqueiros, Portinari, etc. Dicha exposición será instalada en el salón de exposiciones de la planta baja del Museo Nacional" (AHMNC vol. 21 1949, fol. 88).

32 artistas de las Américas se abrió al público entre el 8 y el 17 de febrero de 1949. La selección y gestión de obras y artistas estuvo a cargo del crítico cubano José Gómez Sicre, quien había estado trabajando en la Unión Panamericana desde 1945, primero como especialista de arte y una vez creada la OEA (1948), como jefe de la sección de Artes Visuales. ${ }^{5}$

5 José Gómez Sicre se doctoró en Derecho diplomático en 1941 por la Universidad de La Habana y desde entonces ya era amigo de diversos artistas cubanos del movimiento moderno, lector e incluso al momento de la exposición 
Fue precisamente en los años de 1940 y 1950, como resultado de un proceso que se estuvo gestando desde las primeras décadas del siglo xx, cuando Estados Unidos, y sobre todo los circuitos neoyorquinos encabezados por el MoMA, se procuraron un lugar central en la escena artística de Occidente (Guilbaut). En ese contexto, Gómez Sicre trabajó desde la OEA abriendo un camino a la exportación del modelo de arte moderno abanderado por el MoMA y su institucionalización en Latinoamérica, y lo hizo de una manera más enfática y precisa que la que Lincoln Kirstein, Alfred Barr e incluso Nelson Rockefeller habían empezado a gestionar años atrás.

El contacto constante con las figuras más influyentes del arte en los países americanos fue una de las estrategias que permitió a José Gómez Sicre convertirse no solo en especialista, sino en la principal figura de vínculo entre el arte del sur del continente y los Estados Unidos. Esta dinámica se mantuvo durante su gestión como director del Departamento de Artes Visuales de la OEA (1948-1977), la que ha sido ampliamente cuestionada por estar relacionada con las acciones de intervención colonialista de los Estados Unidos en Latinoamérica durante la Guerra Fría, tal como se hace evidente en los trabajos de historiadores como Shifra Goldman, Alessandro Armato, Claire Fox y Michael Wellen, entre otros.

32 artistas de las Américas puede ser pensada como el comienzo del cuestionado proceso mediante el cual se forjó la institucionalidad para el arte moderno en Latinoamérica en la segunda mitad del siglo xx. En sus lineamientos curatoriales, esta exposición no estuvo muy alejada del evento en el MoMA en 1943. Para el caso de algunos países era lejana, poco precisa y general. Tenía un carácter más diplomático que artístico y, más que nada, daba cuenta de la manera en que se habría coleccionado arte moderno latinoamericano en los Estados Unidos en las dos últimas décadas. Y aunque sin duda se presentaron obras de grandes artistas, a la hora de exponer el acontecer plástico latinoamericano del momento se quedaba corta.

Otros eran sus puntos fuertes, según el texto de exposición de su catálogo, escrito por José Gómez Sicre. El evento enfatizaba en la voluntad de consolidar una comunidad artística panamericana, respaldada en un ideal libertario e independiente: “[...] esta exposición se propone manifestar que en América el hombre puede dejar a su espíritu recorrer todas las categorías y dimensiones de la creación, como corresponde a un continente cuyo primer designio es el de la libertad. Si lograra fijar esta idea, sería ya recompensa suficiente para su misión [...]” (AHMNC vol. 17 1949, pza. 6).

En virtud de su propósito, la exposición fue de carácter itinerante, comenzando en Colombia y pasando por Ecuador, Uruguay, Argentina, Cuba, El Salvador, Chile, Panamá, Puerto Rico, entre otros (AHMAA). Tal como se anotaba en el catálogo, de

de la colección de arte latinoamericano del MoMA en 1943, colaborador de Alfred H. Barr, Jr. Su interés en el arte lo llevó a escribir crítica en su país natal y a organizar diversas exposiciones. Una vez en los Estados Unidos, donde llegó apoyado por Barr, hizo estudios de arte en las universidades de Columbia y Nueva York, lo que le permitió ingresar a la Unión Panamericana con carácter de especialista. Para ampliar los datos biográficos del crítico cubano, revisar Armato (89-97). 
las 32 piezas de arte seleccionadas, 18 pertenecían a la colección de arte latinoamericano del MoMA y las restantes a colecciones públicas y privadas ubicadas en los Estados Unidos. Así, los artistas colombianos que representaron a Colombia fueron Luis Alberto Acuña, con La ciudad dorada, y Gonzalo Ariza con Bogotá, ambas obras para ese entonces consignadas en el museo neoyorquino.

A pesar de no hacer un aporte crítico sustancial estéticamente hablando, al mostrar una evolución mínima desde 1943, la muestra traída desde Washington propició el acercamiento entre José Gómez Sicre y artistas y gestores activos en la región, desencadenando importantes vínculos profesionales y de amistad, que pronto darían frutos. ${ }^{6}$

En Bogotá, el evento panamericanista fue apoyado por diferentes actores, tanto artísticos como diplomáticos, pertenecientes sobre todo a la Embajada de Estados Unidos, el Museo Nacional y la Escuela de Bellas Artes. Vale la pena reseñar el acercamiento que a partir de este evento se generó entre el director de Artes Visuales de la OEA y Alejandro Obregón, quien había empezado a destacar en la escena local como docente y gestor, además de encontrarse en un punto álgido de su carrera artística. Para 32 artistas de las Américas, José Gómez Sicre y Alejandro Obregón solamente se comunicaron por correo, sin embargo, a finales de 1949 se encontraron personalmente en París y gradualmente consolidaron una amistad que duró varios años. En un texto del crítico cubano sobre Alejandro Obregón, relata los inicios de su amistad en París durante 1949 y el mutuo apoyo que caracterizó su relación:

Comencé a tratar al artista, por razones más o menos oficiales, cuando él era director de la Escuela de Bellas Artes de Bogotá, a distancia y por carta. Nos perdimos de vista por una temporada de pocos años y, finalmente, nos encontramos en París, en dos ocasiones: 1949 y 1954. Como era natural, con su poder de persuasión y su alegría vital, me arrastró por distintos lugares [...] Durante veinte años he seguido paso a paso la carrera de Alejandro Obregón (Gómez Sicre, "Alejandro Obregón" 17-18).

Alejandro Obregón se trasladó a Alba-Francia en 1949, desde donde continuó en contacto con José Gómez Sicre. Allí permaneció hasta 1954 y desarrolló una parte sustancial de su obra artística.

Tanto en la trayectoria de Obregón como en la de sus compañeros de generación, si bien Estados Unidos fue un referente obligado sobre todo a nivel de difusión, Fran-

6 José Gómez Sicre empezó a contactar y apoyar a varios artistas a lo largo del continente, que se movían fuera de la línea "mexicanista", entre los que se cuentan Rufino Tamayo y José Luis Cuevas de México; Alejandro Obregón, Enrique Grau y Édgar Negret de Colombia; Carlos Mérida de Guatemala; Rodrigo Peñalba de Nicaragua; Fernando de Szyszlo de Perú; Armando Reverón, Héctor Poleo, Alejandro Otero y Mateo Manaure de Venezuela; Armando Pacheco de Bolivia; Roberto Matta, Pablo Burchard y Carlos Faz de Chile, entre algunos otros. Además de su respaldo para participar en exposiciones en los Estados Unidos, el crítico cubano apoyó la participación de estos artistas en eventos como la Bienal de São Paulo (Armato 109). 
cia, España, Italia, e incluso México y luego Brasil, también lo fueron. Al igual que el MoMA, los salones en la OEA y más adelante el Museo de Bellas Artes de Houston y el Guggenheim, las bienales hispanoamericanas de Venecia, París, São Paulo, México y Córdoba formaron parte del mapa de viaje de los artistas colombianos.

En este sentido, y de acuerdo con Andrea Giunta, vale la pena ofrecer matices a aquella idea tan difundida que propone que después de la Segunda Guerra Mundial el foco del arte moderno occidental se trasladó definitivamente a los Estados Unidos y más precisamente a Nueva York. Es claro que Nueva York tuvo influencia definitiva en el desarrollo del arte moderno a nivel global, sin embargo, los artistas provenientes de América Latina no dejaron de viajar a otros centros artísticos, desde donde también se estaban replanteando las vanguardias, y a partir de sus experiencias, poco a poco se procuraron expresiones propias que podían asimilarse con éxito en contextos internacionales. Podemos decir de manera amplia que no hay una adscripción rígida a un centro artístico, más bien la dinámica mundial propició desarrollos artísticos simultáneos que controvertían la figuración tradicional y de este modo avanzaron modernidades particulares y paralelas (Giunta).

Antes de la partida de Obregón a Alba para dedicarse de lleno a la pintura, desde la dirección de la Escuela de Bellas Artes, función que ejerció entre 1948 y 1949, realizó gestiones relevantes para la comprensión del papel de la modernidad en Colombia: fue uno de los hombres clave en la inauguración de un importante espacio comercial para el arte moderno en Bogotá, las Galerías de Arte, donde además de exponer, colaboró en la organización de exposiciones de arte moderno como parte del cuerpo de asesores técnicos. ${ }^{7}$ Se encargó de organizar eventos que, apoyados por el Museo Nacional, reunieron importantes artistas locales, que a pesar de sus diferencias, coincidían en su voluntad modernista. Dentro de estos eventos podemos reseñar una exposición de pintura contemporánea conocida como el Salón de los Veintiséis en octubre de 1948 y el Salón Nacional de Arte Moderno en noviembre de 1949 (Mesa, ¿Identidad o modernidad? 15-17).

Con el impulso inicial que significó 32 artistas de las Américas, el Departamento de Artes Visuales de la Unión Panamericana emprendía una dinámica que se mantuvo con diferentes intensidades por más de dos décadas. En diciembre de 1949 la OEA presentó en Washington una exposición de 17 artistas contemporáneos de Colombia, en la que a los artistas de Lincoln Kirstein se sumaban Marco Salas Vega, Marco Ospina, Eduardo Ramírez Villamizar, Guillermo Silva, Sergio Trujillo y Francisco Tuminá, entre otros. Unas pocas obras habrían sido prestadas por los artistas y las demás eran parte de la colección de pintura de la norteamericana Mrs. B. Forrest Uhl, directora del colegio Estados Unidos en Bogotá. La exposición, cuyas gestiones

7 Sobre el perfil moderno e internacional de las Galerías de Arte vale la pena consultar: "Extraordinaria labor llevarán a cabo las Galerías de Arte, de esta ciudad", El Tiempo, 23 de julio de 1948. 
estuvieron a cargo de José Gómez Sicre, se inauguró con un protocolo diplomático al que asistieron figuras políticas prominentes. Tras su visita en Washington la muestra fue a Nueva York, Oklahoma, Dallas y Los Ángeles, tal como se anotó en el catálogo correspondiente (AHMAA Colombia 1949).

Esta exposición ofrecía un matiz importante a lo que antes se había acotado como arte colombiano de cara al extranjero, y continuaba apoyándose en el valor individual de las obras y los artistas. Además de "los veteranos" como Ariza y Acuña y los “autóctonos" como Ramírez Fajardo y Tuminá, se presentaba a los “jóvenes” Ramírez y Ospina. Así lo anunciaba Jane Watson Crane en un artículo titulado "Fresh Work up at Pan-American Union", publicado en el Washington Post el 4 de diciembre de 1949:

The show from Colombia reveals originality and initiative. It ranges from the tried and successful results of an accomplished veteran like Luis Alberto Acuña to the spontaneous pen and ink interpretations of tribal lore by an Indian. The group represents individual effort rather than any school or tradition. [...]

Paintings by Gonzalo Ariza convey the mountains green growth and impressive broad plateaus of Colombia with clarity and a complete lack of self-consciousness. Ariza, who is one of the few American artists to have studied in Japan, seems to hear few traces of his oriental training.

Among works by the younger artists are three paintings by Eduardo Ramirez Villamizar and an abstraction by Marco Ospina, evidences in both cases of a break with academic tradition (AHMAA Colombia 1949).

Tras esta exposición vinieron otras tantas, cada vez más alejadas de los lenguajes academicistas, varias de ellas acogidas por la sala de la OEA y otras por diferentes espacios de arte y galerías. Pronto se realizó Sculptures and Paintings of Colombia en el New School for Social Research en Nueva York (1950), donde se expusieron de manera exclusiva obras de Enrique Grau, Eduardo Ramírez y Édgar Negret (fig. 5). Tras otras exposiciones de Negret en el Clay Club, la galería Peridot en Nueva York y la Universidad de Nebraska, esta última adquirió una de sus esculturas para la Frank M. Hall Collection, poniendo al artista colombiano al nivel de los grandes escultores contemporáneos (fig. 6): "Mr. Negret's work joins distinguished examples by Lachaise, Zorach, Marini and Moore in the University's growing collection of modern sculpture" (AHMAA Negret 1950-1951).

A pesar de que el período comprendido entre 1948 y 1951 fue complejo para Colombia en términos políticos y esta condición afectó el apoyo estatal a los circuitos artísticos, el impulso dado por medio del interés latinoamericanista desde la OEA, el naciente espacio de arte instaurado en el Museo Nacional y la voluntad de artistas y otros actores privados, promovió que tanto los lenguajes como los espacios de circulación comenzaran a adquirir rumbos propios. 
FIGURA 5

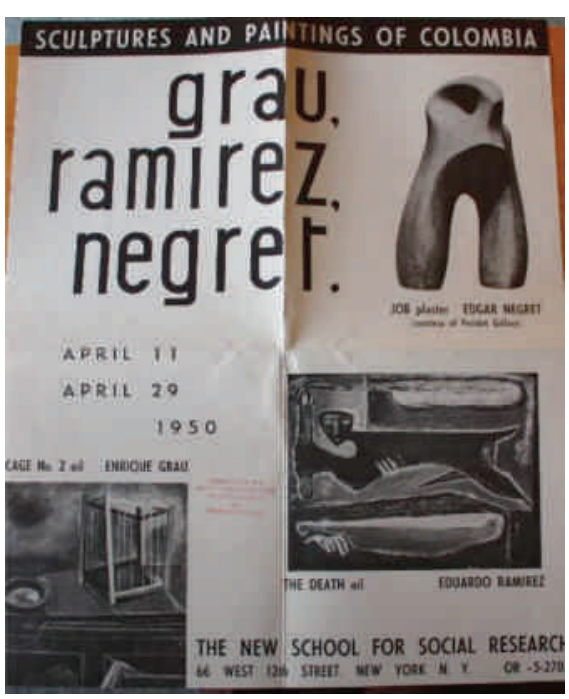

Plegable de la exposición Sculptures and Paintings of Colombia en el New School for Social Reaserch en Nueva York, 1950 (AHMAA Negret 1950).
FIGURA 6

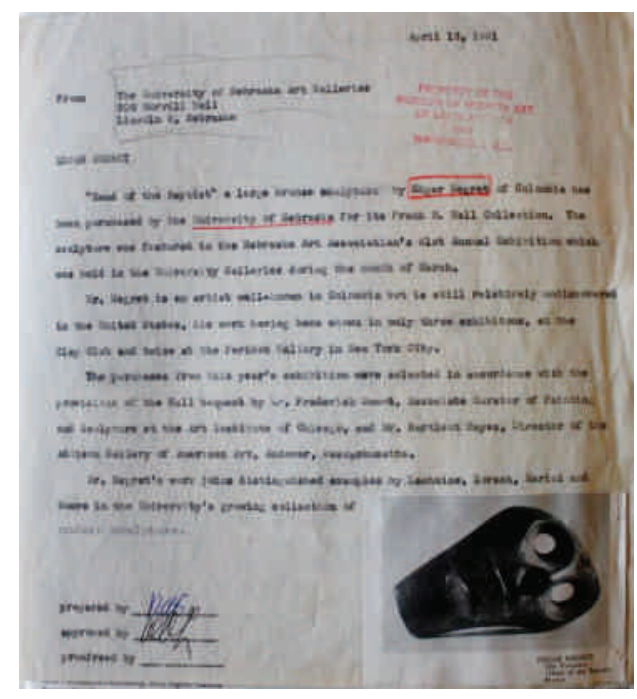

Informe de adquisición de la escultura de bronce, El visionario (cabeza del bautista) de Édgar Negret (AHMAA Negret 1950).

\section{¿Una exposición de arte moderno sin Picasso?: Artistas colombianos en la Bienal Hispanoamericana de Arte (1951-1956)}

[...] Los poseedores de la verdad -oráculos, profetas, poetas y artistas- muy pocas veces hablan un idioma científico y realista. Prefieren las alegorías, las parábolas, las metáforas, los refranes, los mitos y los sueños. Porque, digámoslo con las palabras del propio Picasso, "el arte es una mentira que nos hace conocer la verdad”. Para poder expresar esa verdad, el artista tiene que trabajar con libertad. El presidente Roosevelt, refiriéndose a los países totalitarios, lo dijo bien claramente: "Las artes no pueden prosperar más que allí donde los hombres puedan ser libremente tal como son y disciplinar ellos mismos sus propios ardores y energías [...] La libertad política trae libertad en el arte [...] El que aplasta la individualidad en el arte, aplasta el arte mismo" (Barr, ¿Qué es la pintura moderna? 44).

Otro de los eventos internacionales de los que se hicieron partícipes los artistas colombianos a mitad del siglo xx fue la Bienal Hispanoamericana de Arte, instancia que en sus tres versiones (Madrid 1951-1952, La Habana 1954 y Barcelona 1955-1956), ha sido ampliamente estudiada desde perspectivas múltiples. Investigadores como Miguel Cabañas Bravo, Genoveva Tusell, María Luisa Bellido, Katia Figueredo y Eva 
March Roig, entre otros, se han interesado en asuntos tanto artísticos como políticos y de diplomacia cultural vinculados a esta bienal, teniendo en cuenta que su realización, además de ser fundamental en los procesos de institucionalización del arte moderno en España a mitad del siglo xx, fue una de las acciones más importantes y polémicas de política internacional en torno al arte y la cultura, instaurada por la dictadura franquista durante la década de 1950.

Algunos de los argumentos que se han esgrimido a la hora de analizar este evento de arte han girado en torno a preguntas como: ¿Qué implicaba que una dictadura militar financiara una exposición de arte moderno de grandes dimensiones y con un perfil internacional? ¿Cuál fue la posición de los artistas más independientes en los escenarios internacionales, frente a un evento organizado por un régimen militar y gestionado para salvar relaciones diplomáticas? Esto teniendo en cuenta, por ejemplo, que el arte moderno representado en la figura de Picasso y su Guernica, ya sólidamente avalado por los circuitos artísticos franceses y norteamericanos, había sido exiliado de España con la llegada de la Guerra Civil cerca de quince años atrás.

A mitad del siglo xx, dos asuntos resonaban a la hora de pensar la modernidad artística en Occidente y se identificaban plenamente tanto con la figura como con la obra de Pablo Picasso: "La obra de arte es un símbolo, un símbolo visible del espíritu humano en su busca de la verdad, de la libertad y de la perfección" y "Libres para expresarse, libres de necesidad y libres de miedo es cómo deben sentirse los artistas". Estos dos preceptos básicos, concernientes al perfil ético tanto de la obra como del artista moderno, acotados en 1943 en What is Modern Painting? -y ratificados tras su reedición y traducción al español y al portugués entre 1952 y 1953 y en las múltiples exposiciones del artista español acogidas por el MoMA- planteaban una tensión a la hora de pensar en la pertinencia de la invitación que la España franquista extendía a artistas españoles y americanos. ${ }^{8}$

Las bienales hispanoamericanas son un buen ejemplo de la manera en que los Estados apropiaron la modernidad artística para dinamizar su participación en la geopolítica mundial a mitad del siglo xx. Vale la pena recordar que para este momento, por su afinidad a los regímenes totalitarios de Europa, España estaba bloqueada política y comercialmente, y que la diplomacia cultural fue una de sus estrategias para reconectarse con el mundo. Además del prestigio que otorgaron estos eventos culturales a los participantes, a los países y a las instituciones de arte que los impulsaron, vale la pena pensar en los efectos y las tensiones que sus motivaciones propiciaron en los circuitos artísticos locales, los ejercicios creativos y las subjetividades artísticas.

La Bienal Hispanoamericana, desde su concepción, era inconsistente frente a la libertad creativa, sin embargo, ofrecía a los artistas la posibilidad de tener contacto y

8 Una amplia descripción y análisis de los detalles de la realización de las bienales hispanoamericanas han sido desarrollados por Miguel Cabañas Bravo (La política artística del franquismo; Artistas contra Franco). 
reconocimiento internacional y, en consecuencia, valorizar su obra tanto material como simbólicamente. En ese momento era complicado que un escenario independiente de los aparatos estatales ofreciera una plataforma de visibilidad de dimensiones tan amplias, teniendo en cuenta, entre otras cosas, la inversión que esto requería. ¿Qué podía ser más coherente para un artista que por antonomasia defendía la libertad? ¿Negarse enfáticamente a participar de una bienal concebida a partir de intereses políticos en el contexto de una dictadura militar? $\mathrm{O}$ ¿actuar como un caballo de Troya entrando en la plaza enemiga para apropiarla? En principio la reacción de los más autónomos fue la primera. También hubo figuras de prestigio internacional como Salvador Dalí, quien apoyó plenamente la iniciativa del Estado español y criticó a quienes no lo hicieron:

Por encima de toda política, la España real se encuentra en España. Su imperio espiritual culminó y culminará en sus pintores con el genio de Picasso inclusive, pese a su actual comunismo. Ante la antipatriótica tentativa de Picasso de sabotear nuestra Bienal, ruego a los pintores españoles y americanos de no secundarle. Personalmente tomo las disposiciones necesarias para que mi exposición de Londres sea transportada en bloque a Madrid, a fin de clausurar solemnemente la Bienal, ya que arreglos anteriores me han imposibilitado asistir a su inauguración. Le autorizo la publicación de este telegrama en la prensa, y le saludo muy cordialmente. -Salvador Dalí (cit. en Cabañas Bravo, La política artística del franquismo 309).

Como lo evidencia este comunicado, la tensión ética de participar o no en el evento hispanoamericanista afectó de manera directa a Pablo Picasso, quien tras ser invitado se opuso radicalmente a asistir a la primera y segunda edición, difundiendo manifiestos que desencadenaron en eventos "contrabienales" principalmente en París, Ciudad de México y Caracas para la primera bienal, y en La Habana para la segunda. Paradójicamente -y muy probablemente jugando esta vez a ser caballo de Troya-, para el evento en Barcelona en 1955 Picasso consintió en que, por primera vez después de 15 años, algunas de sus obras fueran expuestas en un evento oficial en España. Del mismo modo, otros tantos artistas españoles y americanos que habían rechazado las dos primeras invitaciones accedieron a asistir a la última bienal, generando un impacto importante a la hora de pensar las vanguardias artísticas en un contexto hispanoamericano.

La posición de los artistas colombianos tuvo matices. La contribución de Colombia al evento oficialista, salvo la edición de La Habana en la que el país estuvo representado por una sola artista, fue numerosa y el gobierno nacional desde el Ministerio de Relaciones Exteriores y el Ministerio de Educación apoyó los envíos. Por otro lado, hubo artistas que manifestaron su desacuerdo e incluso participaron en las acciones contra la bienal motivadas desde París. 
Para la exposición de 1951 en Madrid, Colombia participó con "veinte y tantos artistas" una representación amplia en la que se incluían varias generaciones y estilos. ${ }^{9}$ Para esta edición el eco disidente de Pablo Picasso se manifestó en dos contextos. En primer lugar, Alejandro Obregón, que hacía un par de años se encontraba en Francia, fue uno de los artistas latinoamericanos que se unió a la contrabienal parisina realizada entre diciembre de 1951 y enero de 1952, evento que contó con cerca de cuarenta artistas sumando españoles exiliados y latinoamericanos. Asunto que, como referencia Miguel Cabañas, desde la embajada de España en Colombia se notificó en los siguientes términos:

Como contra partida al éxito que la iniciativa de la Primera Exposición Bienal de Arte Hispanoamericano ha tenido en Colombia; éxito en el que ha participado tanto el mundo oficial como el artístico, algunos elementos -entre los que colean dos o tres exiliados resentidos- han intentado deslucir el triunfo de la concurrencia colombiana a la Bienal de Madrid. Para ello -y sin duda bajo investigaciones recibidas por los centros de exiliados de París- han intentado mover una campaña, para organizar una posible exposición, con otros elementos que los concurrentes a Madrid, en la misma fecha. La iniciativa ha partido, según parece, de un mensaje, que firmado en primer lugar por Pablo Ruiz Picasso, excita a la celebración de exposiciones -por decirlo así- disidentes (Despacho núm. 206 de fecha 19-9-51 del embajador de España en Bogotá. Cit. en Cabañas Bravo, La política artística del franquismo 446).

En sintonía con la acción disidente de Obregón, según anota Miguel Cabañas, hubo quienes manifestaron su inconformidad; sin embargo, al parecer sucedió cuando el gobierno nacional ya habría enviado las obras a Madrid. Otro gesto que además de indicar la falta de convicción en el evento, denotaba falta de confianza en la selección que podría hacerse desde Colombia, se refleja en la carta enviada por Pedro Nel Gómez y Eladio Vélez desde Medellín a las directivas del Museo Nacional, expresando interés de participar con la condición de que no se abrieran las cajas con sus obras en Bogotá, sino que se hiciera un envío a ciegas a Madrid (AHMNC vol. 25 1951, fol. 408). No hay indicio de la forma en que se resolvió el evento y finalmente en el catálogo se registra a Pedro Nel Gómez como parte de la delegación colombiana.

En cuanto a la II Bienal, fue un evento mayoritariamente cubano y español y, como se señaló antes, la presencia colombiana se limitó a una participante, la artista

9 Los artistas colombianos participantes que registra el catálogo general son los pintores Julio Castillo, C. B. Ramos, Blanca Sinisterra, Pedro Nel Gómez, Sergio Trujillo, Darío Tobón Calle, Efraím Martínez, Rafael Sáenz, L. Espada de Valencia (este nombre está equivocado en el catálogo, corresponde a Lucy Tejada de Valencia), Carlos Correa, Luis Acuña, Santiago Martínez Delgado, Luis Ángel Rengifo, C. Tupaz Mejía, Gonzalo Ariza, Eduardo Ramírez Villamizar y Miguel Padilla y los escultores Ramón Barba, Luis Cardona, José Domingo Rodríguez y Guillermo Uribe Aguirre (Cabañas Bravo, La política artística del franquismo 425-426). 
antioqueña Mariela Ochoa. Varios eventos podrían haber incidido en el tamaño mínimo de esta delegación: en primer lugar, el país se encontraba en un momento complejo; cerca de un año atrás se había establecido la dictadura del general Gustavo Rojas Pinilla y, como efecto de la crisis política, la atención a las artes mermó y se detuvieron las gestiones para el Salón Nacional, que solo volvió a realizarse en 1957 cuando se retomó la democracia. Al tiempo, hubo rechazo al evento y se reveló en un manifiesto firmado por algunos artistas: Alipio Jaramillo, Marco Ospina, Jorge Elías Triana, Eduardo Ramírez Villamizar, Ignacio Gómez Jaramillo, Enrique Grau, León Cano y Jorge Moreno Clavijo. En este documento, dirigido a la opinión pública de Cuba, se negaban a participar en una "Exposición Bienal que organiza el gobierno de Franco" y que se hacía "bajo la propaganda de un régimen fascista" (Cabañas Bravo, La política artística del franquismo 446).

Sin duda, un giro importante tanto desde la oficialidad como desde el campo artístico sucedió en el envío colombiano para la edición de Barcelona en 1955. La delegación volvió a crecer y, distinto a la multiplicidad de géneros que se vieron en el pabellón colombiano en el certamen de Madrid, este fue un envío enfocado en valores plásticos actualizados. Participaron 18 artistas, algunos de los cuales se habían negado a hacerlo antes. Esta vez la mayoría presentó obras alejadas de la figuración historicista: Eduardo Ramírez Villamizar, Fernando Botero, Marco Ospina, Francisco Cárdenas, Judith Márquez, Cecilia Porras, Antonio Valencia, Ignacio Gómez Jaramillo, Luis Alberto Acuña, Lucy Tejada y Alejandro Obregón -quien resultó premiado-, entre otros. Además, se otorgó a Colombia el Gran Premio a la Aportación de un País.

En la I Bienal tanto Blanca Sinisterra como José Domingo Rodríguez habían recibido premios de pintura y escultura, respectivamente. La asignación de este galardón estuvo mediada por un jurado nacional compuesto por personalidades de la sociedad colombiana, en su mayoría ajenas a las artes y quienes a pesar de los valores modernos con los que competían (Lucy Tejada o Eduardo Ramírez, por ejemplo), sobre todo para el caso de la pintora, procuraron premiar la belleza academicista. Muy diferente fue lo que sucedió para el envío y la premiación en el evento de Barcelona en 1955 que, aunque paradójicamente se efectuaba en medio de la dictadura de Rojas Pinilla, apostaba hacia las vanguardias. Esto se podría explicar teniendo en cuenta que las dinámicas de los circuitos artísticos locales habían empezado a funcionar con cierta eficacia, a pesar de la irregularidad del salón oficial.

Vale la pena mencionar la impresión que tenían algunos artistas colombianos en relación con la bienal y en general con la escena artística española de la época, evidente en una carta escrita por Lucy Tejada en 1955 y enviada al crítico polaco Casimiro Eiger, quien para ese entonces dirigía la galería El Callejón en Bogotá. Lucy Tejada contaba a su amigo sobre la dinámica escasa de los circuitos españoles sin contar la bienal, sobre la alta calidad de la representación colombiana y las posibilidades que tenía Obregón para obtener un premio (Tejada). 


\section{FIGURA 7}

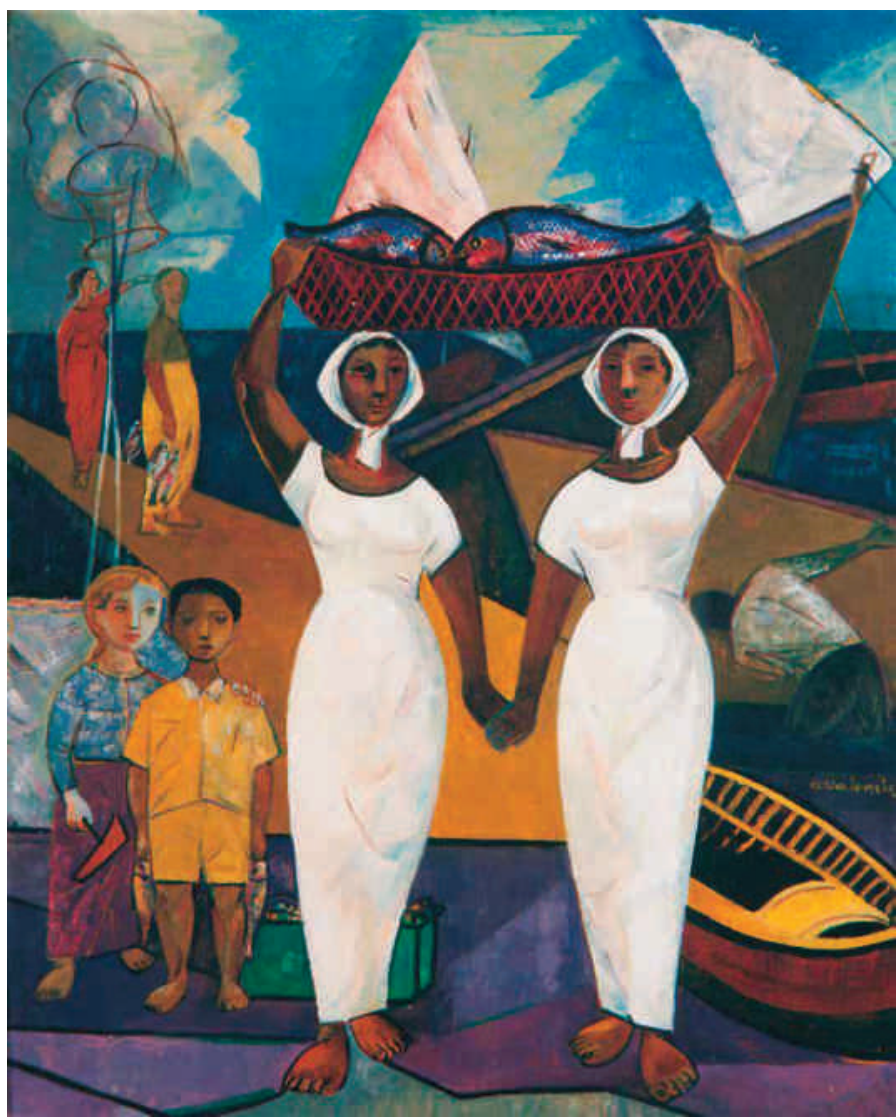

Antonio Valencia, El Puerto (ca. 1955). Óleo sobre tela, 146 x $120 \mathrm{~cm}$. Colección Agencia Española de Cooperación Internacional, n. ${ }^{\circ}$ inv. $205 \mathrm{ICH}$. Reproducción fotográfica (Agencia Española de Cooperación Internacional 426).

A la hora del envío a la III Bienal, Alejandro Obregón se encontraba de regreso en el país, Marta Traba recién había llegado y pronto se insertaría al ejercicio crítico en la prensa y la televisión. Un pequeño circuito comercial empezaba a adquirir fuerza, destacando las actividades de las galerías Buchholz y El Callejón (Serna Lancheros). Desde el Ministerio de Educación se apoyaban exposiciones de artistas actuales en la Biblioteca y el Museo Nacional, y se empezaba a gestionar la fundación de un museo de arte moderno en Bogotá (Mesa, "Museo de Arte Moderno de Bogotá..."). Se considera también el trabajo que desde 1948 venía haciendo el Departamento de Artes Visuales de la OEA para posibilitar la circulación de artistas nacionales en espacios y colecciones internacionales.

En los años siguientes a las bienales hispanoamericanas, ingresaron a la colección de arte del Centro de Cultura Hispánica obras de artistas que habían participado en 
FIGURA 8

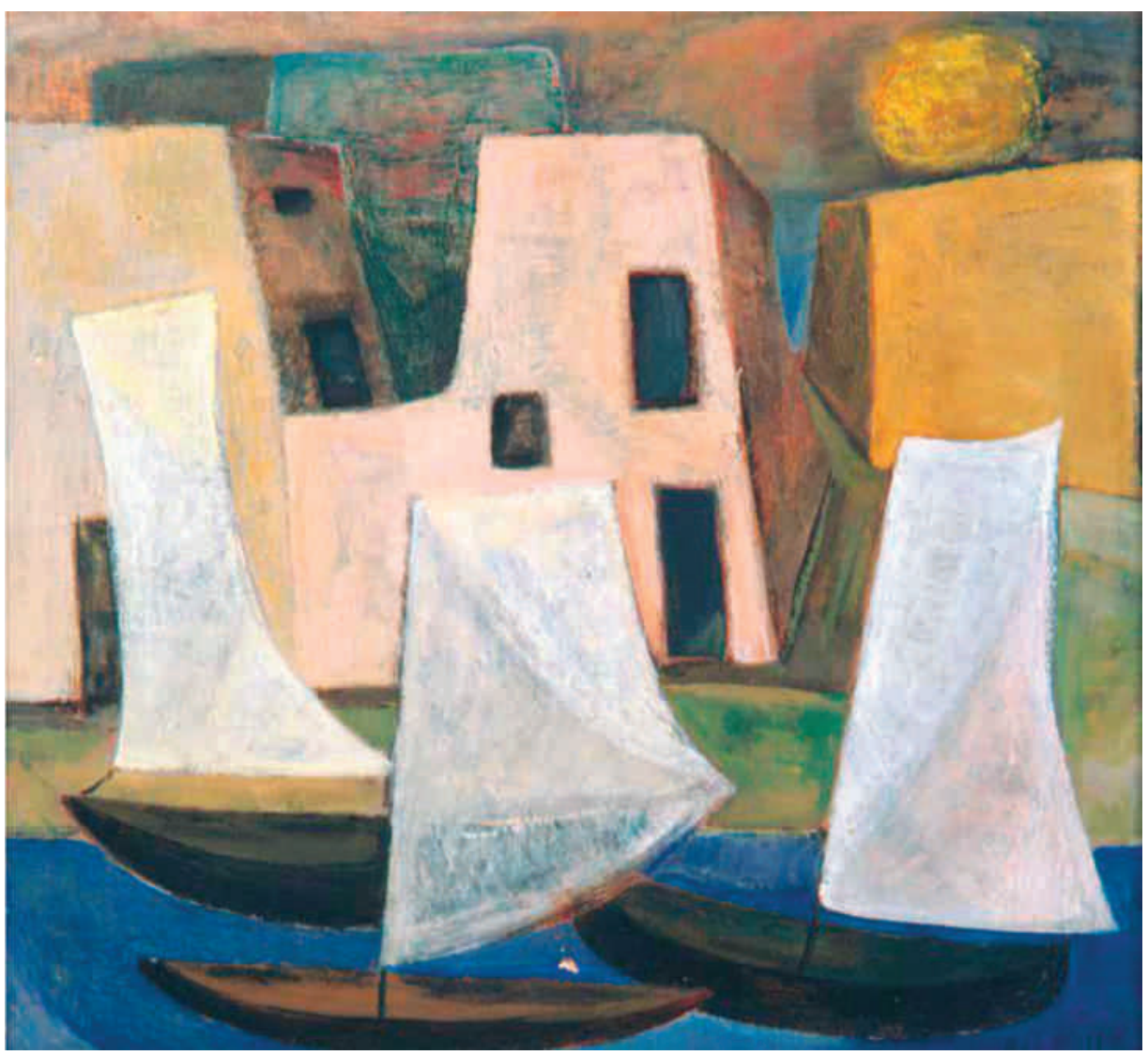

Cecilia Porras, Botes (1955). Óleo sobre tela, 57 x 65 cm. Colección Agencia Española de Cooperación Internacional, n. ${ }^{\circ}$ inv. 64 ICH. Reproducción fotográfica (Agencia Española de Cooperación Internacional 400).

el evento de Barcelona y que daban cuenta del estado actual de la pintura moderna en Colombia como: Antonio Valencia (fig. 7), Cecilia Porras (fig. 8), Fernando Botero (fig. 9) y Alejandro Obregón (fig. 10). Sumado a la actividad en torno a la Bienal Hispanoamericana, para enero de 1956 era numerosa la cuota de artistas colombianos que había pasado por la Sala de Exposiciones de la Unión Panamericana en Washington; además, por medio de su Fondo Interamericano, el MoMA recientemente había adquirido obras de Édgar Negret, Sign for an Aquarium, y de Alejandro Obregón, Souvenir of Venice (MoMAA Recent Acquisitions November 30, 1955-February 22, 1956). 
FIGURA 9

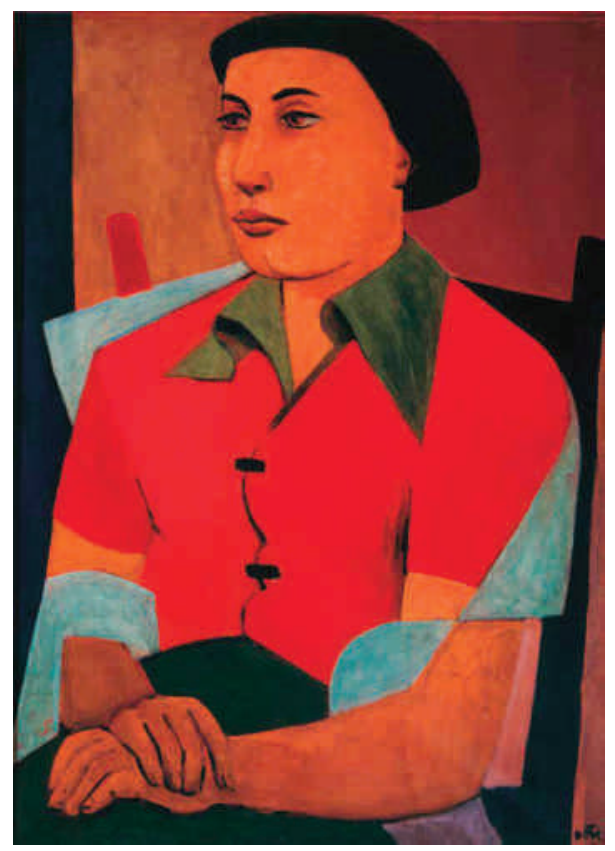

Fernando Botero, Retrato de Brunhild (1954). Óleo sobre tela, 109 x 79 cm. Colección Agencia Española de Cooperación Internacional, n. ${ }^{\circ}$ inv. $64 \mathrm{ICH}$. Reproducción fotográfica (Agencia Española de Cooperación Internacional 277).
FIGURA 10

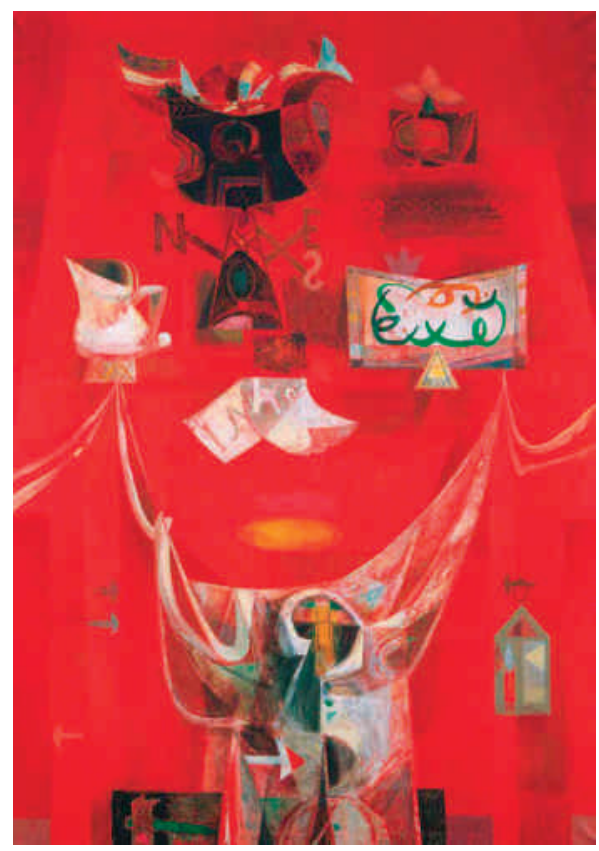

Alejandro Obregón, Mesa del Gólgota (1955). Óleo sobre tela, 161 x 113 cm. Colección Agencia Española de Cooperación Internacional, n. ${ }^{\circ}$ inv. $224 \mathrm{ICH}$. Reproducción fotográfica (Agencia Española de Cooperación Internacional 370).

\section{Conclusión}

Se percibe un tránsito importante entre la discursividad plástica propuesta por Luis Alberto Acuña, Gonzalo Ariza, Erwin Kraus y Alfonso Ramírez Fajardo y asumida como moderna por el MoMA en los primeros años de la década de 1940, y las propuestas artísticas de mediados de los cincuenta que representaron al arte colombiano en eventos como la III Bienal Hispanoamericana. Ser moderno en Colombia cada vez estaba más relacionado con las posibilidades de interacción internacional, en busca de aquel "esperanto" que habría anunciado Alfred Barr en su momento. El reto era entonces lograr este registro universalista sin dejar de reflexionar sobre asuntos propios.

1956 fue un año particularmente fructífero en términos de internacionalización para los artistas colombianos y particularmente para Alejandro Obregón. Tras el cierre de la bienal de Barcelona en enero, entre otros eventos, en agosto desde el Museo Nacional y con el aval del ICOM se convocó a los artistas locales a participar en la primera edición de los premios Guggenheim. Un par de meses después se anunció a Obregón como ganador del concurso a nivel nacional, con su pintura Velorio, y se gestionó el envío de esta junto a obras de otros cuatro artistas al gran evento inter- 
nacional a realizarse en París. Al terminar el concurso, la OEA compró Velorio para su colección y el Museo de Bellas Artes de Houston adquirió Ganado ahogándose en el Magdalena, que también había merecido reconocimiento internacional en el Gulf Caribbean Competition. Como ya se ha anotado, este fue el contexto en que el Museo Nacional decidió adquirir Máscaras.

La apuesta estética de estas tres pinturas, que salieron del fuero privado del artista para, tras su reconocimiento internacional como pinturas modernas, entrar a ser parte de colecciones públicas, dista ampliamente del academicismo y de cualquier tipo de pintura historicista o folclórica, apuntando a reflexiones vigentes como el colonialismo y la violencia.

Sin duda, las dinámicas culturales en tiempos de Guerra Fría posibilitaron al arte latinoamericano -y con él al colombiano- hacerse partícipe de escenarios internacionales de circulación, ingresar a colecciones de arte moderno alrededor del mundo y, a que gradualmente los acervos públicos al interior del país adquirieran obras, reconociendo tanto su valor artístico como patrimonial.

El reto para los artistas era complejo: debían conservar la autonomía creativa y reflexionar sobre sus propios asuntos; conseguir un ejercicio plástico acorde a los procesos de internacionalización para el arte vigentes, al tiempo que participaban de un sistema de circulación afectado por las tensiones políticas y éticas de un mundo polarizado.

Finalmente, este texto abre posibilidades para la comprensión de la discursividad artística colombiana de mitad del siglo xx, por medio del análisis de prácticas de circulación que afectaron tanto al perfil del artista moderno como al de la obra de arte y la manera como esta ha sido asimilada, apropiada y resignificada con el paso de los años.

Apostar por estructurar las investigaciones desde perspectivas metodológicas alternas, en este caso historiando prácticas de circulación y coleccionismo, permite matizar la historia de los procesos artísticos más allá de la genialidad de sus artífices y de las opiniones de los críticos, posibilitando relaciones complejas entre los discursos plásticos y sus contextos, y alcanzando reflexiones que van más allá de adscripciones políticas subjetivas.

\section{Referencias}

Adams, Beverly. "The Challenges of Collecting Latin American Art in the United States: The Diane and Bruce Halle Collection". Collecting Latin American Art for the 21st Century. Ed. Mari Carmen Ramírez. Houston, Museum of Fine Arts, 2002, pp. 156-177.

Agencia Española de Cooperación Internacional. Colección Artística de la Agencia Española de Cooperación. Ed. María Conde Blanco. Ministerio de Asuntos 
Exteriores y de Cooperación - Agencia Española de Cooperación, 2016. http:// bibliotecadigital.aecid.es/bibliodig/es/catalogo_imagenes/grupo.cmd?path=1011953 Visitado 13 de febr. de 2018.

AHMAA. Archivo Histórico Museo de Artes de las Américas. Washington, 1948-1956. AHMNC. Archivo Histórico Museo Nacional de Colombia. Bogotá, 1943-1956.

Armato, Alessandro. "La influencia de Nelson Rockefeller, el MoMA y la OEA en la construcción de São Paulo como foco de irradiación para el arte moderno en Latinoamérica: Los casos del MAM-SP y de la Bienal (1946-1959)”. Tesis de maestría en Historia del arte argentino y latinoamericano, I-UNSAM, 2014.

Barr, Alfred Hamilton. "Foreword". The Latin-American Collection of the Museum of Modern Art. Lincoln Kirstein. New York, The Museum of Modern Art, 1943, pp. 3-4.

---. What is Modern Painting? New York, The Museum of Modern Art, 1943.

---. ¿Qué es la pintura moderna? Trad. Luis de Zuleta. New York, The Museum of Modern Art, 1953.

---. La definición del arte moderno. Ed. Irving Sandler. Trad. Gian Castelli. Madrid, Alianza Editorial, 1989.

Basilio, Miriam. "Reflecting on a history of collecting and exhibiting work by artists from Latin America”. Latin American \& Caribbean art: MoMA at El Museo. Ed. Miriam Basilio et al. New York, El Museo del Barrio \& The Museum of Modern Art, 2004, pp. 52-68.

Bellido Gant, María Luisa. "Derroteros del arte latinoamericano en España”. Arte latinoamericano de siglo XX: Otras historias de la historia. Ed. Rodrigo Gutiérrez Viñuales. Zaragoza, Prensas Universitarias de Zaragoza, 2006, pp. 263-284.

Cabañas Bravo, Miguel. Artistas contra Franco: La oposición de los artistas mexicanos y españoles exiliados a las bienales hispanoamericanas de Arte. México, D. F., Universidad Nacional Autónoma de México - Instituto de Investigaciones Estéticas, 1996.

---. La política artística del franquismo: El hito de la Bienal Hispano-Americana de Arte. Madrid, Consejo Superior de Investigaciones Científicas, 1996.

Figueredo Cabrera, Katia. "Francisco Franco y Fulgencio Batista: complicidad de dos dictadores en el poder (1952-1958)”. Tzintzun. Revista de Estudios Históricos 64, 2016, pp. 296-325.

Fox, Claire F. Making Art Panamerican: Cultural Policy and the Cold War. Minneapolis, University of Minnesota Press, 2013.

Giunta, Andrea. “Transatlánticos / Transcontinentales. Una discusión sobre diálogos y genealogías”. Encuentros transatlánticos: discursos vanguardistas en España y Latinoamérica. Museo Nacional Centro de Arte Reina Sofía. Madrid, 11 al 13 de julio de 2013. 22. Abstracts. http://www.museoreinasofia.es/sites/default/files/ actividades/programas/abstracts_congreso_0.pdf Visitado 12 de febr. de 2018.

Goldman, Shifra. Contemporary Mexican Painting in a Time of Change. Texas, University of Texas Press, 1981. 
Gómez Sicre, José. “Alejandro Obregón”. Americas, Washington DC, julio-agosto 1983, pp. 17-18.

---. 32 artistas de las Américas: exposición organizada por la Unión Panamericana. Bogotá, Museo Nacional de Colombia, 1949.

González, Beatriz y Cristina Lleras. Colección de pintura: Museo Nacional de Colombia. Bogotá, Planeta, 2004.

Guilbaut, Serge. De cómo Nueva York robó la idea de arte moderno. Valencia, Tirant lo Blanch, 2007.

Kirstein, Lincoln. The Latin-American Collection of the Museum of Modern Art. New York, The Museum of Modern Art, 1943.

March Roig, Eva. "Franquismo y vanguardia: III Bienal Hispanoamericana de Arte". Espacio, Tiempo y Forma, serie VII, 3, 2015, pp. 33-54. <http://revistas.uned. es/index.php/ETFVII/article/view/12036>. Visitado 13 de febr. de 2018.

Mesa, Alexandra. “ ¿Identidad o modernidad?: Colección de Arte del Museo Nacional de Colombia (1948-1963)”. Inédito, 2017.

---. “Museo de Arte Moderno de Bogotá primera pincelada, 1962-1965”. Quintana 15, 2016, pp. 203-222.

MoMAA. The Museum of Modern Art Archives: Exhibition History. New York, 1943-1956. 1002 2018. <https://www.moma.org/calendar/exhibitions/history>. ---. "Lincoln Kirstein Correspondence and Notes". Museum of Modern Art Archives. 1940-1944. <https://www.moma.org/learn/resources/archives/EAD/Kirsteinf>.

Ornelas Cota, Eustáquio. "A formação de coleção latino-americana do Museu de Arte Moderna de Nova York: cultura e politica (1931-1943)”. Tesis de maestria em Historia social, Faculdade de Filosofia, Letras e Ciências Humanas da Universidade de São Paulo, 2016.

Pacheco, Marcelo. "New Art Collecting Trends in Argentina: the 1990s". Collecting Latin American Art for the 21st Century. Ed. Mari Carmen Ramírez. Houston, Museum of Fine Arts, 2002, pp. 99-129.

Pérez Oramas, Luis. "The Art of Babel in the Americas". Latin American \& Caribbean art: MoMA at El Museo. Ed. Miriam Basilio et al. New York, El Museo del Barrio \& The Museum of Modern Art, 2004, pp. 34-51.

Sandler, Irving. "Introducción”. La definición del arte moderno. Alfred H. Barr, Jr. Trad. Gian Castelli. Madrid, Alianza Editorial, 1989.

Serna Lancheros, Julián Camilo. "El valor del arte. Historia de las primeras galerías de arte en Colombia (1948-1957)”. Ensayos. Historia y teoría del arte 17, 2009, pp. 61-84.

Tejada, Lucy. "A Casimiro Eiger”. Madrid, 1 de noviembre de 1955. Proyecto Archivo Digital y Publicaciones del Museo de Bellas Artes de Houston. <http://icaadocs. mfah.org/icaadocs/ELARCHIVO/RegistroCompleto/tabid/99/doc/1094204/ language/es-MX/Default.aspx> Visitado 13 de febr. de 2018. 
Traba, Marta. “Las generaciones de la Escalinata”. El Tiempo, 7 de julio de 1957. http:// www.eltiempo.com/archivo/documento/CMS-12716042. Visitado 16 de ene. de 2018.

Tusell García, Genoveva. "Picasso, A Political Enemy of Francoist Spain”. The Burlington Magazine 155.1320, 2013, pp. 167-172.

Wellen, Michael. "Pan-American Dreams: Art, Politics, and Museum-Making at the OAS, 1948-1976”. <https://repositories.lib.utexas.edu/handle/2152/ETDUT-2012-12-6625>.Tesis doctoral, Universidad de Texas, 2012.

Zalamea, Jorge. Nueve artistas colombianos. Bogotá, Litografía Colombiana, 1941.

Enviado: 08 marzo 2018 Aceptado: 10 septiembre 2018 